\title{
Basal cisternostomy - Is it a panacea for traumatic brain swelling?
}

\author{
I. Cherian
}

Professor and Chief, Department of Neurosurgery, College of Medical Sciences, Bharatpur, Chitwan, Nepal

\begin{abstract}
The evolution of modern neurosurgical techniques in traumatic brain injury has been ongoing for the last two centuries. However it has always been a challenge to obtain an effective clinical outcome, especially those following severe traumatic brain injuries. Other than the well established procedures for acute and/ or chronic subdural hematomas and depressed skull fractures, newer avenues for development of surgical techniques where indicted have been minimal.
\end{abstract}

Practical scenario in trauma neurosurgery comes with multiple challenges and limitations. In an emergency setup, primary management of traumatic brain injuries fall upon the on-duty resident or medical officer in training. Due to the emergent nature of the condition and time being an important variable, the experience of the operating surgeon as well as the severity of the injury become an important contributing factor in the disease prognosis. Based on clinical experience and observation of acute neurosurgical service in tertiary medical center in a developing country, a novel technique in the management of trauma neurosurgery have been elucidated in the current study. The study proposes to apply the principles of microvascular surgery and skull base surgery in selected cases of severe traumatic brain injuries.

Key words: Basal cisternostomy, brain swelling, head trauma.

\section{Introduction}

Traumatic brain injury (TBI) is a major public health concern worldwide with no significant change in its epidemiology over the last thirty years. ${ }^{1}$ The burden of death or disability from TBI has been increasing worldwide. According to the World Health Organization report TBI is expected to surpass many other diseases, such as ischemic heart disease and cerebrovascular disease, as a major cause of death and disability by the year $2020 \mathrm{AD}^{2}$ The outlook in trauma neurosurgery in most centers worldwide has not made significant progress in the past several decades. The most

Correspondence: I. Cherian

E-mail: drrajucherian@gmail.com significant step in this direction has been the introduction of decompressive hemicraniectomy and supportive management in an intensive care unit (ICU) with pressure monitoring. This may be attributed partly to poor prognosis of the disease related to its severity as well as practical circumstances surrounding the scenario such as inexperienced surgeons or peri and intra-operative care facilities in different centers. In addition, trauma neurosurgery has been mostly looked upon as an extension of the general trauma team and therefore less appealing to aspiring neurosurgeons who aim to be trained in skull base, vascular or spinal surgical specialties. Furthermore, interventions for trau- 
matic intracranial lesions and or raised intracranial pressure have been fairly standard, eg. medical management of raised intracranial pressure or ventricular catheterization, decompressive craniectomy, surgical evacuation of hematoma etc. ${ }^{3}$ Owing to these factors, advancing research and opportunities for improvement in surgical techniques in the field has been close to nonDepending on surgical indication in a given case scenario, it is imperative that patients be managed with optimal care. Thus the research and improvement of surgical technique in trauma has remained to be almost nonexistent while non surgical ways of treating traumatic brain injury is fast gaining popularity. Research in conservative management of traumatic brain injury with therapeutic options for neuroprotection has been rigorously pursued over the last forty years. ${ }^{4}$

It is important that an appropriate surgical algorithm and technique be applied to the management of traumatic brain injury, where indicated, for better patient outcome. Therefore in the current study, a novel technique which incorporates the discipline of skull base and microvascular approach of opening the sub arachnoid cisterns has been described. Furthermore, it has been recommended that the technique can be reproduced in any well equipped tertiary care center where the on-duty neurosurgery consultant is adequately trained and is cognizant of the pathophysiology of trauma neurosurgery and its approach to management. It is note-worthy that the microvascular approach described in this study has been similar to previously well described cases of aneurysmal sub-arachnoid hemorrhage and related surgical procedures. ${ }^{5-8}$

\section{Materials and methods}

The present study has been initiated from 2009 and is continuing till date in CMS, Bharatpur.
In the present study pure extradural hematomas have been excluded as it is a condition which improves on evacuation and we do not intend to modify the current technique. We classify the other causes of head injury as 1. Unilateral single contusion with mild to moderate mass effect (with or without subdural hematoma) 2. Unilateral multiple contusions with brain swelling (with or without subdural hematoma); 3. Unilateral subdural hematoma with mass effect; 4. Unilateral brain swelling with no appreciable lesion (with or without subdural hematoma); 5. Bilateral contusions and bilateral subdural hematomas with brain swelling. Although there are other combinations which were seen, more than 90 percent of the operated cases fell into either one of these categories.

We would like to discuss the management of these scenarios in the following section as has been done at ourinstitution

\section{Case scenario 1}

A large bone flap based on the size of the contusion from pre-op CT image is planned. This is followed by a $\mathrm{C}$ shaped dural opening based on the sylvian. The initial dural opening should be small and made as basal as possible. This could be enlarged later on by incisions perpendicular to the original incision. The purpose of this maneuver is to access the basal region and let out CSF and blood at high pressure after which there would be space for retraction and reach the basal cisterns. In case of brain swelling, the sylvian can be readily accessed and other measures as mentioned below could be done. At the earliest hint of brain swelling, we open the cisterns and do the steps mentioned below for the case scenarios 2,3,4. The operative field is continuously irrigated. In case of a superficial contusion, the clot drains by itself, and if the contusion is within the brain parenchyma the pia needs to be in- 
I. Cherian, Basal cisternostomy - Is it a panacea for traumatic brain swelling?

cised with simultaneous instruction to the anesthetic team for a Valsalva maneuver while the contusion is being evacuated gently. Use of bipolar cauterization is not recommended unless in case of an arterial bleeder. For venous ooze, application of surgical and gentle placement of cotton patties is advised. This is followed by irrigation for approximately 5 minutes. In our experience the bleeding has been found to stop by this maneuver.

\section{Case scenario 2, 3 and 4}

A large fronto-parieto-temporal trauma flap measuring approximately $13 \mathrm{~cm}$ anterioposteriorly is made. The inferior limb of the flap extends upto the zygoma. Removal of the sphenoid ridge upto the superior orbital fissure (SOF) is done although there is no need to meticulously expose the SOF. This bone work helps access the cisterns even in a tight brain where retraction or any undesired manipulation is not recommended. A ' $C$ ' shaped dural opening is made on the proximal part of the sylvian fissure starting from the most basal part of the dura exposed. It is important to have a gush of CSF mixed blood as soon as the basal dura is opened. This phenomenon is encountered in almost all the cases. After the dural opening, the sylvian is gently punctured in multiple points using a 24 gauge needle and these tears are made confluent with microscissors. This contributes to CSF egress making the brain becomes sufficiently lax for the next step.

Subfrontal retraction using a wide brain spatula is done as the next step in a very gentle fashion. The olfactory nerve helps in leading one to the optic nerve and the interoptic cisterns can be opened by gentle suction. After CSF starts to flow out, the brain becomes much more lax and opening of the optico carotid, lateral ca- rotid and interhemispheric cistern is performed. The cisterns on the opposite side also can be opened. Once the brain is e adequately retracted, the lamina terminalis can be opened. This is followed by opening of the membrane of Lilliquest after widening of the optico carotid window or the lateral carotid window. The visualization of the basilar artery and the pons ensures that most of the CSF spaces are opened. The cisterns are gently irrigated for any residual blood clots. Adequate hemostasis is maintained by application of surgicel cut into small squares and continous irrigation. This is followed by closure performing a duroplasty and replacement of the bone after biting away the temporal part.

\section{Case scenario 5}

Procedure described for case scenario 2,3 and 4 is performed bilaterally if there are hematomas on both side with significant mass effect. Otherwise a unilateral approach to opening both sided cisterns may be employed.

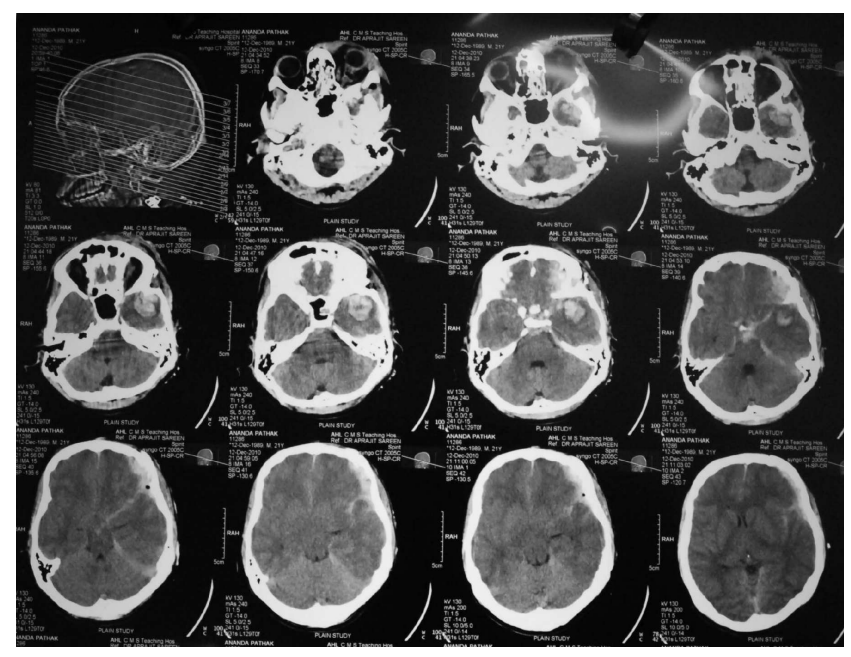

Fig 1: A Show the preoperative CT scan of a male patient brought to the Emergency with a GCS of 8. The left temporal contusion, frontotemporal subdural, extensive subarachnoid blood in the cisterns and severe brain swelling may be noted. 


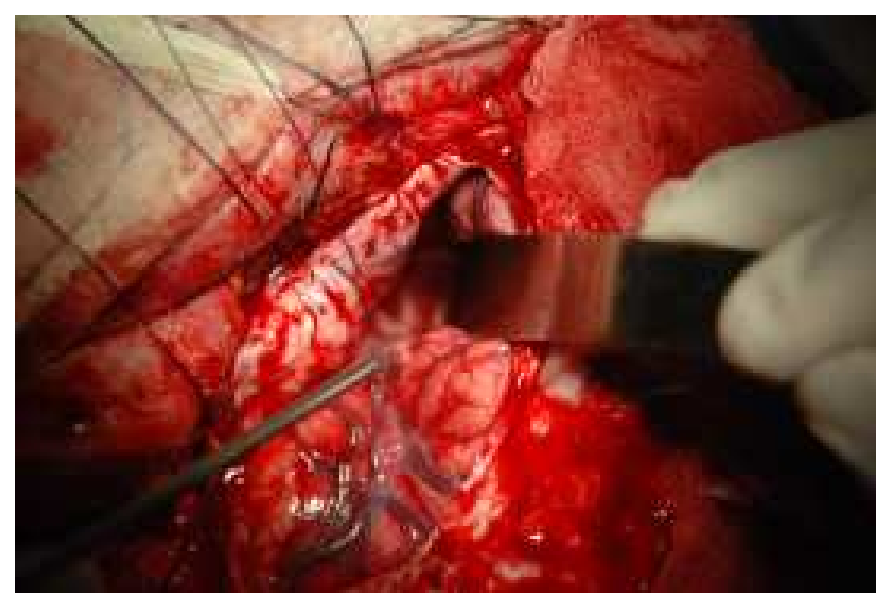

Fig. 1 B: Intra operative picture of the brain showing the optic nerves bathed in CSF. This is despite the preoperative CT scan showing slit ventricles and compressed cisterns. We have tried to explain the reason in our discussion.

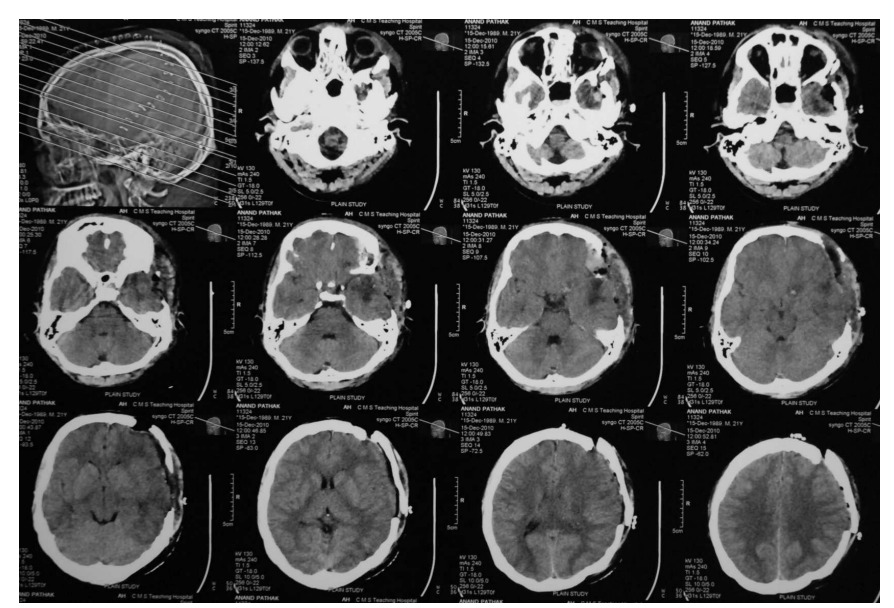

Fig 1 C: Post operative scans of the same patient who was $15 / 15$ in 3 days.

\section{Results}

Over the past 28 months we managed 211 cases of traumatic brain injury with definitive indication for neurosurgical intervention. 43 patients were operated for purely extradural hematomas and hence were excluded from the study. 74 patients were diagnosed as unilateral contusions with or without a subdural hematoma with significant mass effect. Hence, these patients fell under the classification of case scenario 1.49 patients had unilateral multiple contusions with or without subdural hematoma and significant mass effect and hence was classified as case scenario 2.34 cases had unilateral subdural hematomas with mass effect and were classified as case scenario 3. Seven cases had predominantly unilateral brain swelling sometimes with a small accompanying subdural hematoma and these were classified as case scenario 4 . Four cases had bilateral contusions and bilateral subdural hematomas with brain swelling.

After excluding the extradural hematomas, according to GCS there were 35 mild head injuries (GCS 13 15), 87 moderate head injuries (GCS 9 to 13) and 46 severe head injuries (GCS 3-9).

There were no mortalities in the mild head injury group. 4 patients died in the moderate head injury group ( 4.5 percent) and 6 patients died in the severe head injury group (13 percent).

\section{Discussion}

It is to be remembered that most of the CSF resides in the cisterns and cisternal opening helps decrease the raised intracranial pressure (ICP) substantially. The surgical approach we have proposed is based on the following strategic and presumed mechanism. Please note that this is only our hypothesis and it may require years of lab work and clinical correlation to prove it.

We would like to divide the intra cranial pressure into 1. intra arachnoidal pressure and 2. intra brain pressure. These are usually kept in equilibrium by fluid shift presumably through the Virchow Robin spaces (VR spaces) and the bulk flow of CSF 
I. Cherian, Basal cisternostomy - Is it a panacea for traumatic brain swelling?

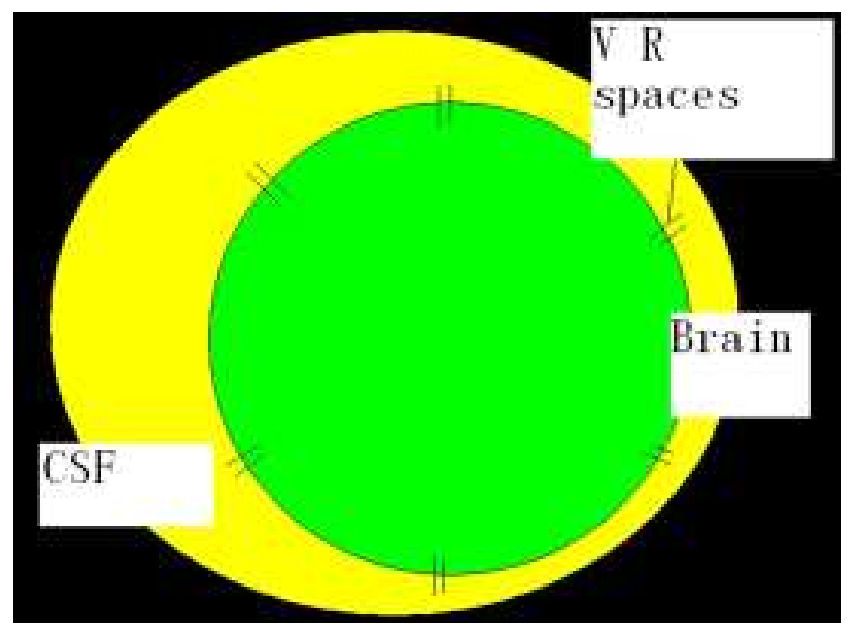

Fig. 2A: In the normal scenario, the inta - Brain pressure (I will call this IBP) and the intra cranial pressure (ICP) are almost equal and there is transport of fluid thru Virchow Robin spaces between the brain and the CSF.

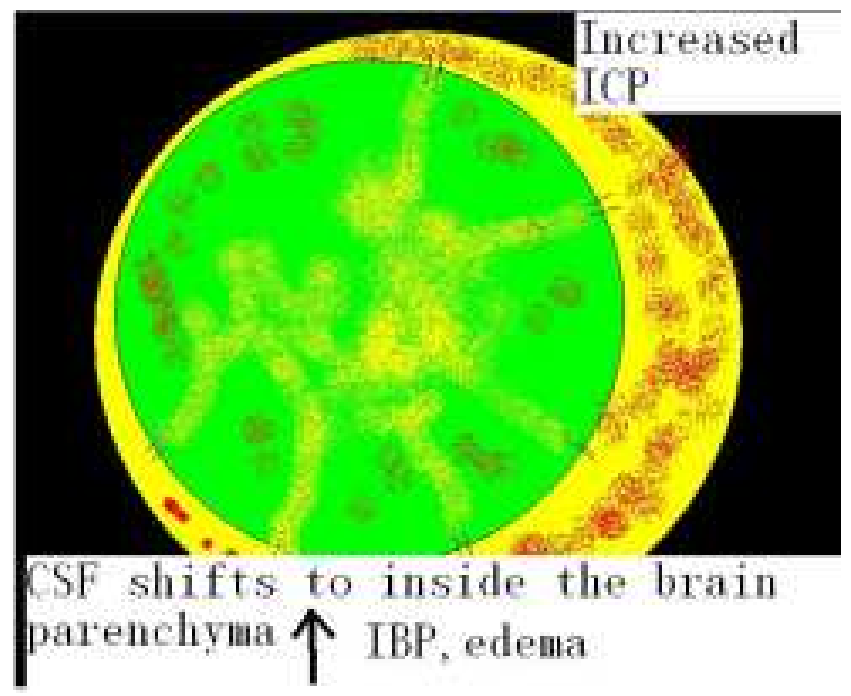

Fig. 2B: In trauma there is bleeding into the subarachnoid and subdural spaces. (In frank hematoma of the brain, the hematoma has to be drained to decrease the intra brain pressure. But in cases with subdural hematoma, subarachnoid hematoma and severe brain swelling the intra arachnoidal pressure increases and there is fluid shift into the brain which is the cause for sudden and severe brain edema)

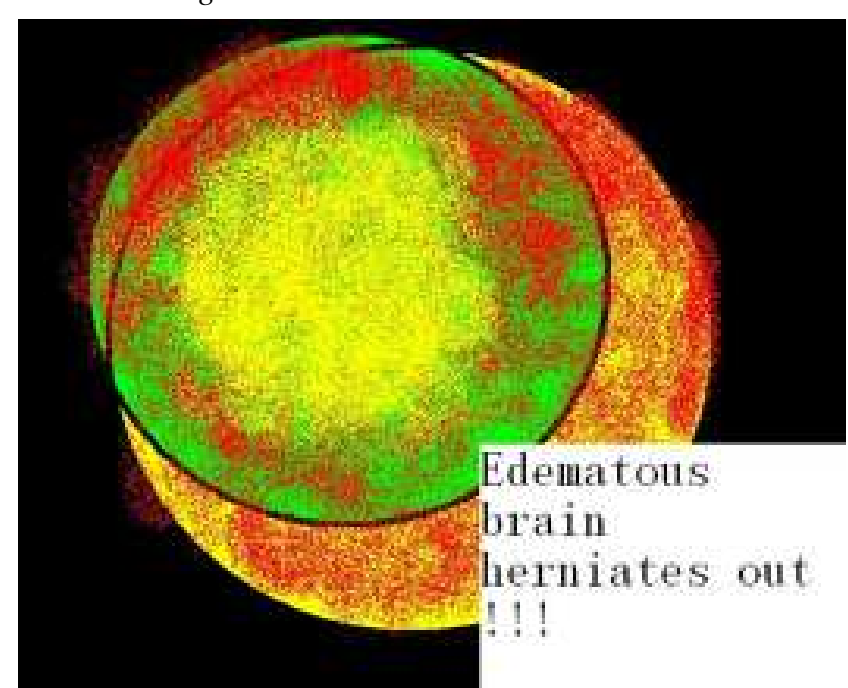

Fig. 2C: So the intra cranial pressure increases .. this will lead to a CSF shift into the brain through Virchow Robin Spaces and this will cause increased intra brain pressure (IBP). This is what we term edema. So, opening the dura in a place where there is no CSF causes the brain to herniate out... and as the pressure decreases after removal of some brain, further shift of fluid into the brain from the CSF compartment takes place and the brain just keeps on coming out.

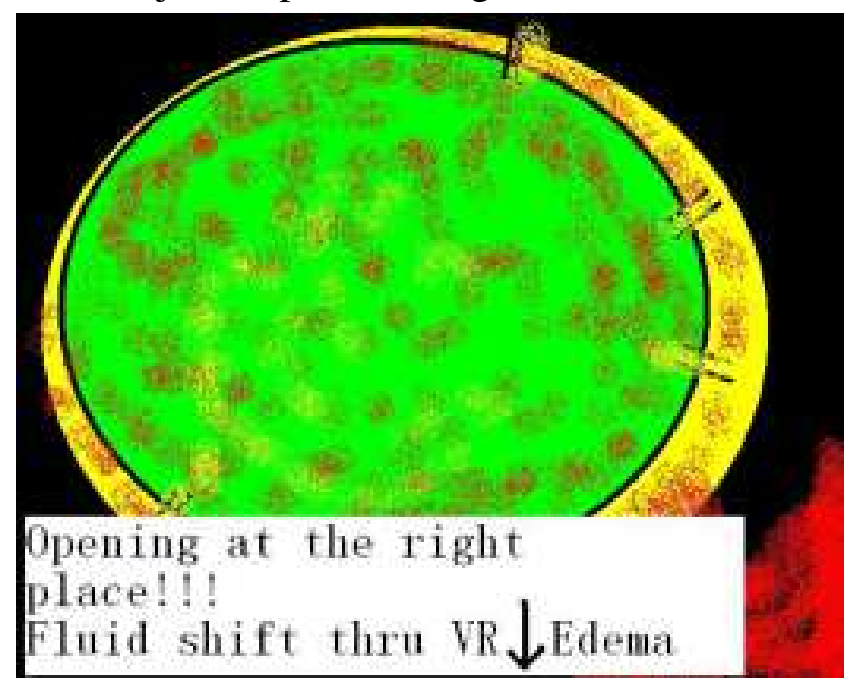

Fig 2D: Opening the basal dura yields blood and CSF at high pressure in almost all the cases. This causes decrease in the intra arachnoidal pressure and there is fluid shift back from the brain to the intra arachnoidal compartment. This results in a lax brain and this allows progressive retraction and opening of all the cisterns upto the membrane of Lilliequist. 


\section{Conclusion}

The practice of opening cisterns has not been practiced in trauma before in a routine fashion. This is an easy technique and can easily be learned by the junior surgeons. This does not require any additional resources and after the learning curve, mortality in head trauma may be reduced in a dramatic fashion. However, the proof for the mechanism may take years of lab work and clinical correlation and to substantiate this as a panacea for diffuse brain swelling, it may require multiple center work and years of close observation.

\section{Acknowledgement}

This work has been dedicated to and in fond memory of late Ms. Mingwei Tan (University of Cambridge, U.K), for her commitment to patient care, contribution to the unit in Nepal during her elective posting in our Department and her dream to be a neurosurgeon. The whole team mourns her and remembers the time that this amazing lady was with us.

Discussions with various senior Neurosurgeons like Dr. Garnette Sutherland and Dr. Sanju Lama, University of Calgary was very helpful in proposing the mechanism. Dr. Ghuo Yi from Baoding was very helpful with discussions regarding the VR spaces.

Disclosure: There is no conflict of interest or any financial or professional disclosure.

\section{References}

1. J.D. Corrigan, A.W. Selassie, J.A. Orman. The epidemiology of traumatic brain injury. J Head Trauma Rehabil. 2010;25(2):72-80.

2. C.J. Murray, A.D. Lopez. Evidence-based health policy-lessons from the global burden of disease study. Science. 1996;1;274(5288):740-3.

3. L.M. Li, I. Timofeev, M. Czosnyka, et al. Review article: The surgical approach to the management of increased intracranial pressure after traumatic brain injury. Anesth Analg. 2010 ;111(3):736-48.

4. R. Vink, M.R. Bullock. Traumatic brain injury: Therapeutic challenges and new directions. Neurotherapeutics. 2010;7(1):1-2.

5. M. Sindou. Favourable influence of opening the lamina terminalis and lilliequist's membrane on the outcome of ruptured intracranial aneurysms. A study of 197 consecutive cases. Acta Neurochir (Wien). 1994;127(1-2):15-6.

6. R.J. Komotar, D.K. Hahn, G.H. Kim, et al. Efficacy of lamina terminalis fenestration in reducing shunt-dependent hydrocephalus following aneurysmal subarachnoid hemorrhage: A systematic review. clinical article. $J$ Neurosurg. 2009;111(1):147-54.

7. R.J. Komotar, A. Olivi, D. Rigamonti, et al. Microsurgical fenestration of the lamina terminalis reduces the incidence of shunt-dependent hydrocephalus after aneurysmal subarachnoid hemorrhage. Neurosurgery. 2002;51(6):1403,12; discussion 1412-3.

8. M. Akyuz, R. Tuncer. The effects of fenestration of the interpeduncular cistern membrane arousted to the opening of lamina terminalis in patients with ruptured $\mathrm{ACoA}$ aneurysms: A prospective, comparative study. Acta Neurochir (Wien). 2006;148(7):725,3; discussion 731-2. 